\title{
Campaña "Fa xwi: Te convido a cuidarnos": Promoviendo la participación de los hombres en la salud sexual y reproductiva*
}

\author{
"Fa xwi or I invite you to care" campaign: promoting \\ young indigenous men's participation \\ in sexual and reproductive health
}

\author{
Nicholas T. Kaufmann ${ }^{* *}$ \\ Universidad Autónoma de San Luis Potosí, San Luis Potosí, México. \\ Luis Gerardo Ayala-Real ${ }^{* * *}$ \\ Organización Salud y Género Querétaro A.C., Santiago de Querétaro, México.
}

Recibido: 6 de febrero de 2018. Aprobado: 22 de marzo de 2018 .

DOI: $10.25100 /$ lamanzanadeladiscordia.v13i1.6707

Artículo de investigación

Resumen: El presente articulo describe el proceso de desarrollo de una campaña de mercadeo social para promover la participación de los hombres jóvenes indigenas en la salud sexual y reproductiva. Llevado a cabo en México, este proyecto aplicó un enfoque intercultural con el fin de identificar los problemas e intereses de jóvenes rural-indígenas y urbano-universitarios. A partir de un diagnóstico participativo, se elaboraron materiales de campaña basados en el uso de conceptos y nociones culturalmente relevantes para la población indígena local. Las lecciones aprendidas del desarrollo de la campaña están abordadas con recomendaciones para futuras acciones.

Palabras clave: Jóvenes; salud sexual y reproductiva; derechos; interculturalidad.

\footnotetext{
*El presente artículo describe el proceso de desarrollo de una campaña para promover la participación de los hombres jóvenes indígenas en México en materia de salud sexual y reproductiva. Llevado a cabo en octubre del 2015, este proyecto aplicó un enfoque de investigación participativa con el fin de elaborar materiales de campaña basados en el uso de conceptos y nociones culturalmente relevantes para la población indígena local. Las lecciones aprendidas del desarrollo de la campaña están abordadas con recomendaciones para futuras acciones.

Agradecimientos: A las y los jóvenes líderes participantes de la comunidad de San Ildefonso Tultepec, y zona de Chiteje, especialmente a la; Lic. María Lucia Pascual Blas y el Est. Noel Cruz Concepción, ambos del Municipio de Amealco, del Municipio de Toliman y de la Comunidad Universitaria de los Estados de Querétaro y de San Luis Potosí. A las autoridades del proyecto Cultura de la Universidad Autónoma de Querétaro. A la Socióloga Mayte Solís González, coordinadora del Encuentro y de la Campaña, a la Dra. Gisela Sánchez Díaz de León, Presidenta de Salud y Género Querétaro AC, al Esp. Hernando Hernández Nava, a la Mtra. Liliana Gutiérrez Leal y al Mtro. Manuel Edmundo Ramos Gutiérrez. A Corazonar AC, y en especial a la Soc. Carolina Cervantes por su participación con una ponencia y la presentación de la exposición fotográfica " 100 años de la paternidad en México".

**Profesor-Investigador de la Facultad de Psicología, Universidad Autónoma de San Luis Potosí, con Doctorado en Investigación Comunitaria y Preventiva por la Universidad de Illinois en Chicago (E.U.A) y Maestría en Psicología Clínica por la Universidad de Ginebra, Suiza. Sus líneas de investigación y acción son: la implementación y evaluación de proyectos de promoción de salud integral con jóvenes desde la perspectiva de género. Correo electrónico: nicholas.kaufmann@uaslp.mx.

***Activista y educador en Salud. Director de proyectos en Salud y Género Querétaro AC. Profesor invitado al posgrado en Familias y Prevención de la Violencia de la Facultad de Ciencias Políticas y Sociales de la Universidad Autónoma de Querétaro, México. Sus líneas de investigación y acción son: Género, masculinidades, salud y la violencia; procesos educativos amplios para la prevención, y para la incidencia en políticas públicas de igualdad de género. Correo electrónico: gerardinoh46@gmail.com.
} 
Abstract: This paper describes the development process of a social marketing campaign to promote young indigenous men's participation in sexual and reproductive health. Based in Querétaro, Mexico, this project applied an intercultural approach to identify issues and interests among indigenous rural and urban-university youth. Following a participatory needs assessment, campaign materials were designed based on the use of culturally relevant concepts and notions for the local indigenous population. Lessons learned from the campaign development process are discussed with recommendations for future actions.

Keywords: Youth; sexual and reproductive health; rights; interculturality.

\section{Planteamiento del problema}

En México, las evidencias epidemiológicas de las últimas décadas (Instituto Nacional de Estadística y Geografía (INEGI), 2013; López, 2013) sugieren que los derechos sexuales y reproductivos no están garantizados para todas las mujeres, y menos para las mujeres jóvenes indígenas que viven en zonas altamente marginadas de la República. Para ellas, no es una realidad el derecho a decidir de manera libre e informada cuando y cuántos hijos tener, el ejercicio de una sexualidad libre de violencia y discriminación, sin riesgo de ITS y VIH/ SIDA o embarazos no deseados; tampoco se respeta el derecho de las mujeres jóvenes indígenas a vivir una maternidad libre, gozosa y segura.

Según la Encuesta Nacional sobre la Dinámica de las Relaciones en los Hogares (ENDIREH), casi la mitad de las mujeres mexicanas mayores de 15 años que han vivido en pareja (47\%) reportan haber sido víctimas de alguna forma de violencia. Mientras que una de cada cuatro mujeres reporta haber sufrido violencia física al menos una vez en su vida y una de cada seis mujeres encuestadas reporta haber sido víctima de violencia sexual al menos una vez en su vida. Según la ENDIREH, ser víctima de las violencias física y sexual es aún más común entre las mujeres hablantes de lengua indígena (HLI) que entre las mujeres no indígenas. Además, del total de las mujeres HLI que reportaron violencia conyugal, 78\% declararon padecer violencia emocional, $45 \%$ económica, $26 \%$ física y $13 \%$ sexual (citado en INEGI, 2013). Las tasas nacionales de violencia de género hacia mujeres HLI son solo un reflejo de la problemática a nivel del estado o del municipio.
Por lo tanto, es importante especificar las condiciones de las localidades en donde esta situación problemática prevalece para mejor entender las maneras de solucionarla.

Este trabajo presenta la problemática existente en el Estado de Querétaro, lugar donde sus autores han colaborado en materia de acciones para la paz, la interculturalidad y la democracia. En las últimas décadas, se ha documentado una disminución demográfica considerable de la población indígena del Estado de Querétaro. Entre 2000 y 2010, este grupo poblacional disminuyó de $3.4 \%$ a $1.8 \%$ del total de la población del Estado. Una serie de indicadores referidos al empleo, ingresos, consumo, vivienda, alimentación, salud y educación colocan a las y los habitantes HLI en claras condiciones de desigualdad y pobreza en comparación a la población no indígena del Estado. Al mismo tiempo, los municipios con mayor presencia de personas HLI en el Estado son considerados de alta y muy alta marginación (ejemplo: Amealco de Bonfil, Tolimán Pinal de Amoles entre otros) por los rezagos en infraestructura básica y productiva, alcanzando apenas un índice de desarrollo humano de 0.7332 . Como principal efecto de estos fenómenos de pobreza, migración y deterioro del medio ambiente, las mujeres son fuertemente afectadas, pues se quedan al cuidado de las familias, tienen que trabajar asalariadamente, cuidar a los hijos, hacer las tareas domésticas, viven sobreexplotación y exceso de responsabilidades que le predisponen a ansiedad, tensión (stress), y baja autoestima, junto con la mala nutrición que agrava su precaria situación de salud.

Las y los jóvenes siguen siendo las personas que menos acuden a los servicios de salud, algo aún más marcado con los hombres jóvenes. Estos suelen buscar ayuda cuando ya están presentes tanto las consecuencias de las violencias como del sexo no protegido. La dimensión sexual y reproductiva aparece más claramente en los egresos hospitalarios en el caso de las mujeres: en el 2009, 350 niñas de 10 a 14 años tuvieron un embarazo. Las tazas en los municipios indígenas son mayores a la tasa estatal y nacional (Servicios de Salud del Estado de Querétaro, 2010).

En el cuidado de la salud sexual y reproductiva se entremezclan los factores para el uso (o no) de mé- 
todos anticonceptivos, asociado no sólo al desconocimiento de los métodos, sino a cuestiones relativas a la cultura y a la educación de género. Sabemos que la población que no tiene satisfecha la demanda de anticonceptivos son precisamente las adolescentes y de ellas, todavía más las que viven en zona rural. El desconocimiento y la falta de una adecuada educación para la salud sexual (especialmente con enfoque de derechos sexuales y reproductivos) favorece que sean jóvenes quienes tienen relaciones sexuales sin saber cómo prevenir una ITS, VIH o embarazo. Esto implica la necesidad de involucrar a los hombres jóvenes en materia de salud sexual y reproductiva para garantizar el derecho de las mujeres a una vida libre de violencia.

El presente trabajo parte del principio de que los hombres son parte de la solución a nivel local y global para prevenir y responder a la violencia sexual y embarazo en la adolescencia. Su propósito es presentar una estrategia comunitaria con perspectivas de género e intercultural para generar en los hombres HLI una reflexión crítica y propositiva en torno al ejercicio de la masculinidad dentro de sus localidades.

\section{Justificación}

Existe una necesidad urgente y no satisfecha para involucrar a los hombres y los niños como aliados en la salud y los derechos sexuales y reproductivos de las mujeres (Fransen-Dos Santos, 2009). De igual importancia, las propias necesidades de los hombres deben de ser atendidas a través de los servicios de salud sexual y reproductiva (Sonfield, 2004).

La falta de involucramiento de los hombres, especialmente de orientación heterosexual, en materia de salud sexual y reproductiva influye negativamente en la salud de las mujeres y las niñas, limitando sus decisiones de vida (Barker y Verani, 2008). A nivel mundial, los hombres usan métodos anticonceptivos a un grado mucho menor que las mujeres. Según cifras recientes de la ONU (2015), sólo el $27 \%$ de hombres en comparación a $73 \%$ de mujeres ha recurrido al uso de anticonceptivos. Por una parte, es importante incrementar la participación de los hombres en el uso de anticonceptivos, promoviendo el uso del condón y la vasectomía, además de métodos de anticoncepción femeninos. Por otra parte, los servicios de salud deben promover que los anticonceptivos sean disponibles y accesibles a los hombres.

En muchas partes del mundo, los hombres siguen dominando las decisiones acerca de cómo y cuándo tener relaciones sexuales, así como la forma y el momento de tener hijos dentro de una relación heterosexual. Dentro de las comunidades indígenas, es probable que exista una concepción de la sexualidad que parte de usos y costumbres más arraigados en los cuales las mujeres no toman decisiones sobre su propio cuerpo, al volverse esposas se convierten propiedad no solo del marido, sino de la familia de este. Además, en contextos rurales los hombres suelen preferir un mayor número de hijos que las mujeres. De hecho, el tamaño de la familia se aprecia a menudo como una medida de la masculinidad. Estas normas basadas en el sistema patriarcal pueden tener consecuencias físicas, emocionales y económicas negativas para las mujeres, así como en los mismos hombres. Así mismo, los embarazos no planeados durante la adolescencia y las infecciones de transmisión sexual, incluyendo el VIH/SIDA, tienen altos costos para las y los habitantes HLI de zonas de alta marginalidad.

Por lo tanto, es importante generar estrategias con las comunidades indígenas que convoquen a los hombres para que participen de manera constructiva en la resignificación de las nociones de masculinidad problemáticas en las que la hombría se asocia a una virilidad percibida. Tales estrategias deben de reconocer a los hombres como aliados ante las mujeres en temas como la planificación familiar y la elección de métodos anticonceptivos.

Tanto el gobierno como las organizaciones de la sociedad civil deben de reconocer que los derechos de todas las personas pueden promoverse mediante programas educativos y campañas de mercadeo social que fomenten en los hombres en particular una reflexión acerca de las formas de vivir la hombría (masculinidades) para reducir y erradicar la violencia de género. Con el motivo de generar esta conciencia, la Alianza MenEngage ${ }^{1}$ lanzó en el año 2014 una iniciativa global dirigida específicamente

\footnotetext{
${ }^{1}$ MenEngage es una alianza global de ONGs y agencias de la ONU que promueve la participación de los niños y hombres en la lucha por la equidad de género. Para más información véase: http://menengage.org/.
} 
a las redes de organizaciones civiles en América Latina que trabajan con hombres como aliados en la promoción de la Salud y los Derechos Sexuales y Reproductivos (SDSR). Esta iniciativa tuvo como meta fomentar a nivel local, regional o nacional propuestas de campañas de mercadeo social para promover SDSR, a través de acciones que:

1. Incrementan el aporte de los hombres a la anticoncepción (uso de condón), y sensibilización para la vasectomía.

2. Aumentan el apoyo de los hombres al acceso y uso de todos los servicios de salud reproductiva para las mujeres.

3. Incrementan la demanda de los hombres y uso de servicios de prevención del VIH, pruebas y tratamiento.

El presente trabajo describe un proyecto que responde a la convocatoria de la región Latinoamericana de la Red Global de MenEngage. Este proyecto fue propuesto desde México por la organización Salud y Género Querétaro A.C. para entender e incidir en las masculinidades indígenas a través de una amplia alianza entre organizaciones de la sociedad civil e instituciones universitarias.

\section{Marco teórico}

Frente a las realidades reflejadas a través de las evidencias epidemiológicas en relación con la violencia de género en el país, el reto más grande según la Organización Panamericana de la Salud en las últimas décadas ha sido cambiar el paradigma de un enfoque de atención a daños de la salud al de estilos de vida sana (Santos-Preciado, Villa-Barragán, García-Avilés, León-Álvarez, Quezada-Bolaños y Tapia-Conyer, 2003). Este cambio de paradigma implica sobre todo una liberación de las representaciones que enajenan y encierran a las personas en comportamientos nocivos para la salud. Con este cambio de paradigma se espera promover prácticas de autocuidado (Arenas-Monreal, Jasso-Arenas y Campos-Navarro, 2011) entre las personas para salvaguardar sus derechos a una vida libre de violencia.

Algunas iniciativas en materia de salud sexual y reproductiva perciben todavía a la juventud como fuente de daños a la salud, como calamidad social que hay que "combatir con buenas maneras". Esta perspectiva estigmatizadora trae consecuencias riesgosas, pues el etiquetamiento peyorativo de las personas facilita la construcción de una identidad negativa (Erikson, 1974). Por lo tanto, las y los jóvenes deben ser vistos como sujetos con derechos, con recursos personales, y no sólo como personas con déficit o como el problema en la sociedad. Sólo a partir de un juicio exterior, una mirada social, que sea fuente de gratificaciones narcisistas (Kiss, Ramon y Sudres, 2004), los estilos de vida sana en adolescentes pueden promoverse.

En México, ya existen varios programas de atención a la salud de las y los adolescentes, en los cuales el enfoque de estilos de vida sana se está manejando. Uno de éstos es el Programa H/M/D: Trabajando en Equidad y Salud con Mujeres y Hombres Jóvenes, desarrollado por la Alianza $\mathrm{H}^{2}$. Este programa, el cual ha sido implementado en México por Salud y Género A.C. ${ }^{3}$, tiene el objetivo de influir la forma en que las y los jóvenes valoran y cuidan su vida, su cuerpo, su salud, tomando como ejes de trabajo la sexualidad y las relaciones afectivas.

El Programa H reconoce la importancia de utilizar una perspectiva ecológica (Bronnfenbrenner, 1979) para reforzar los mensajes asimilados a través de intervenciones a distintos niveles contextuales. Además de talleres educativos dirigidos a determinados grupos de edad, se ha comprobado que las campañas de mercadeo social promueven entre las y los jóvenes un estilo de vida más equitativo en términos de género y a la vez que previenen el VIH / ITS y las violencias (Promundo, Instituto PAPAI, Salud y Género y ECOS, 2013). Entre las experiencias más emblemáticas del Programa $\mathrm{H}$ está la campaña de mercadeo social, llamada la Hora $\mathrm{H}$, que fue diseñada e implementada en contextos de vulnerabilidad social en Brasil. En estos contextos, se reclutó a un grupo de hombres jóvenes con actitudes más equitativas dentro de la comunidad para que trabajen como "promotores pares" en el Pro-

${ }^{2}$ La Alianza H es una iniciativa binacional (Brasil-México) que se basa en el trabajo de cuatro organizaciones no gubernamentales: Instituto Promundo, Instituto Papaï, ECOS y Salud y Género A.C.

${ }^{3}$ Salud y Género A.C. es una asociación civil, conformada por mujeres y hombres de distintas profesiones y experiencias de trabajo que se combina para desarrollar propuestas educativas y de participación social innovadoras en el campo de la salud y el género. 
grama H. A través de su participación, se desarrolló y se implementó una campaña para promover entre los hombres jóvenes el uso de condones y otras conductas más equitativas de género. El proceso de desarrollo de la campaña tuvo éxito debido a la siguiente forma estructurada con la que se llevó a cabo: 1) se realizó un diagnóstico participativo para entender factores asociados a la falta de uso de los condones entre hombres de la comunidad; 2) diseño de materiales de mercadeo social con mensajes culturalmente relevantes para la comunidad; y 3 ) se difundieron los materiales de la campaña a través de diversos canales de comunicación estratégicos en la comunidad (Promundo, Instituto PAPAI, Salud y Género y ECOS, 2013).

$\mathrm{La}$ experiencia del Programa $\mathrm{H}$ indica la importancia de considerar a la población a la que se dirigen las intervenciones a lo largo del proceso de desarrollo e implementación del proyecto comunitario. De igual manera, revela los beneficios asociados con un trabajo en red donde participan diferentes segmentos de la población, inclusive los líderes de la comunidad. Por último, la experiencia subraya la importancia de promover cambios en los estilos de vida a través de mensajes de relevancia cultural en vez de basar la campaña en estereotipos negativos.

Basándose en la experiencia del Programa $\mathrm{H}$, los autores del presente artículo pretenden ilustrar una manera de trabajar desde un enfoque participativo para desarrollar una estrategia integral que busca entender e incidir en las masculinidades indígenas para mejorar la salud sexual y reproductiva de las mujeres indígenas. La población a la que va dirigida esta estrategia está compuesta por miembros de la comunidad Otomí o Ñhoñho, ya que representan hasta un $40 \%$ de la población indígena del Estado de Querétaro.

Esta estrategia consiste en el desarrollo de una campaña de mercadeo social que presenta temas y medios apropiados para difundir mensajes culturalmente relevantes. Para este fin, se describe un ejercicio de diagnóstico participativo, el cual brinda la oportunidad de intercambiar ideas y aclarar muchas dudas respecto a los temas en cuestión. A raíz de este ejercicio, cada punto debe de ser considerado cuidadosamente y debatido dentro del marco gen- eral de los objetivos de la campaña por todos los miembros del grupo organizador junto con las y los jóvenes de la comunidad indígena a la cual se dirigen los mensajes.

Este proyecto propone contribuir a impulsar una amplia alianza entre Organismos de la Sociedad Civil; Red de feministas y diversos colectivos de la comunidad LGBTTI, el Proyecto Cultura de la Universidad Autónoma de Querétaro y el Instituto Intercultural Ñhoñho, en torno al desarrollo de una estrategia de construcción colectiva para la Promoción y Difusión de la Salud Sexual y Reproductiva de Mujeres y Hombres jóvenes, con énfasis en el involucramiento de hombres y enfoque de Interculturalidad y Derechos.

\section{Objetivos}

Con base en una experiencia de investigaciónacción en el campo de la promoción de los derechos y la salud sexual y reproductiva entre jóvenes, el presente trabajo tiene tres objetivos:1) Describir los procesos relacionados al desarrollo de una campaña de mercadeo social; 2) Presentar los materiales de la campaña y los canales de difusión; 3) Identificar las lecciones aprendidas de la campaña, además de recomendaciones para su seguimiento. A continuación, se describe el proceso relacionado al desarrollo de la campaña.

\section{Método}

El desarrollo de la campaña $\mathrm{Fa}$ xwi: Te convido a cuidarnos, es un reflejo de un ejercicio de participación social y reflexión colectiva entre los actores sociales del proyecto que fue informado por elementos del Modelo de Intervención Participativa (Nastasi, Varjas, Schensul, Silva, Schensul y Ratnayake, 2000) que centraliza la cultura en el estudio de las creencias, comportamientos y resultados de salud. Además, este modelo coloca a la cultura en el centro del desarrollo, implementación y evaluación de las intervenciones en materia de salud. De acuerdo con Nastasi et al. (2000), "el foco de la investigación es comprender y describir los fenómenos desde la perspectiva de la población que se estudia, lo que facilita el desarrollo de la teoría e intervención específicas de la cultura" (p. 212). Para este proyecto, mientras que los propios integrantes de la 
organización civil Salud y Género Querétaro A.C (SyGQ) planearon sus primeros pasos, otros actores sociales participaron activamente en el desarrollo de la campaña. Estos pasos coincidieron con tres objetivos que fueron propuestos desde un enfoque intercultural:

a) Realizar un diagnóstico participativo con jóvenes provenientes de dos ámbitos: rural-indígenas y urbanos- universitarios.

b) Elaborar materiales de campaña con lenguaje y conceptos de relevancia cultural.

c) Devolver y difundir la campaña en diversos espacios locales.

\section{Fases de desarrollo de la campaña}

El desarrollo del proyecto se dividió en tres etapas principales: I) diagnóstico participativo, II) elaboración de materiales, III) difusión de la campaña.

\section{I) El diagnóstico participativo: mesas de trabajo}

La realización del diagnóstico participativo se llevó a cabo a través del Encuentro Juventud, Salud y Derechos, un evento colectivo que tuvo lugar el día 17 de octubre de 2015 en las instalaciones de la Universidad Autónoma de Querétaro.

Esta actividad tuvo como objetivo alentar la interacción y el intercambio de experiencias con jóvenes de ámbitos diferentes, alrededor del tema de la Salud y los Derechos, para el desarrollo de una campaña de promoción y difusión de la Salud Sexual y Reproductiva de Mujeres y Hombres jóvenes, con énfasis en el involucramiento de hombres.

El encuentro, contó con la participación de 131 jóvenes entre 16 y 25 años, 47 pertenecientes a la Comunidad Ñhoñho de los municipios de Toliman y Amealco y 84 Universitarios/as de la Autónoma de San Luis Potosí, la Marista, del Tecnológico Regional, la Cuauhtémoc y de la Autónoma de Querétaro, la diversidad de las/los participantes fue su característica más importante y constituyó un aspecto central a considerar en los siguientes momentos de la campaña. El uso de las tecnologías y de las redes sociales, favoreció el proceso de organización del evento, facilitando la movilización de hombres y mujeres jóvenes de diversas procedencias.
El encuentro permitió dialogar y reflexionar acerca de la "Salud y los Derechos" buscando sobre todo que las y los jóvenes tengan oportunidad de expresar sus necesidades y preocupaciones. Este ejercicio se llevó a cabo a partir de una mirada muy amplia de la salud que incluyó la experiencia subjetiva (vida emotiva) y que problematiza el rol de los hombres en las tareas de cuidado (Boff, 2002) cotidianas en diversos entornos familiares y comunitarios. Con este enfoque, el encuentro se centró en cuatro áreas temáticas:

1) Autocuidado y Salud Reproductiva, 2) Violencias del Sistema, Feminicidios y Trata de Personas, 3) Paternidad, Familias y Cuidado, 4) La Diversidad Sexual. Estas áreas temáticas se trabajaron en mesas de trabajo en base a una breve exposición de un/a especialista en el tema para abrir el diálogo y las reflexiones de manera participativa y amplia. Posterior al encuentro, se llevó a cabo la sistematización y el análisis de las principales ideas y los sentires recuperados de las y los jóvenes urbano-universitarios y rurales-indígenas.

\section{II) Elaboración de materiales: diálogo intercultural con comunidad rural-indígena}

La elaboración de materiales para la campaña se realizó con un pequeño grupo de trabajo de ocho líderes jóvenes de la comunidad Ñhoñho y seis activistas de SyGQ, en una reunión llevada a cabo en el Instituto Intercultural Ñoñho, ubicado en el Municipio de Amealco. El proceso de elaboración tuvo como objetivo principal cuidar la interculturalidad del diálogo en la construcción de frases y nociones, así como en la producción de imágenes para la campaña en forma de carteles.

\section{III) La devolución y difusión}

El proyecto se centró en la devolución en por lo menos tres presentaciones públicas y a través de un ejercicio virtual, aprovechando la tecnología y las redes sociales, para alcanzar a muchos otros/ as jóvenes, tanto participantes del encuentro, como otros/as que no participaron. El proceso de difusión consistió en la grabación de spots y la presentación pública y virtual de los carteles. 


\section{Resultados}

Los resultados del proyecto se presentan por etapas. Primero, las principales necesidades, problemáticas y demandas, así como recomendaciones, expuestas por las y los participantes del Encuentro Juventud, Salud y Derechos. Segundo, los materiales de la campaña Fa xwi: Te convido a cuidarnos, que fueron elaborados con frases y nociones culturalmente relevantes para las comunidades Otomí. Por último, se describe los canales que fueron encontrados para la campaña de difusión amplia.

Análisis descriptivo del diagnóstico participativo

Los resultados del diagnóstico participativo se presentan a continuación, según los temas de cada mesa de trabajo. En cada mesa de trabajo, las y los participantes identificaron los principales factores asociados con la violencia hacia determinados grupos poblacionales. Así mismo, se generaron recomendaciones estratégicas para incidir sobre los problemas identificados.

Mesa de Trabajo 1: Salud mental, sexual y reproductiva de niñas-os, adolescentes y jóvenes

El grupo de participantes de la primera mesa de trabajo identificó varios problemas en relación con la salud mental, sexual y reproductiva de niñas-os, adolescentes y jóvenes, los cuales incluyen la deficiente información y conocimiento del cuerpo, el propio y el ajeno, los tabús que reproducen culpa y vergüenza de los asuntos del cuerpo, y las nociones judeocristianas y machistas que dificultan el conocimiento de sí mismo/a. A raíz del diálogo sobre esta problemática, el grupo de participantes propuso las siguientes recomendaciones presentadas en la Tabla 1:

Tabla 1. Recomendaciones para promover la salud mental, sexual y reproductiva en jóvenes

- Campañas de difusión amplia (radio, TV, y redes sociales), con información en materia de salud mental, sexual y reproductiva con base en evidencia, datos e investigaciones científicas, que promuevan el autocuidado, para alcanzar autonomía y autodeterminación para la toma de decisiones informadas, responsables y conscientes.

- Desarrollar programas educativos de sensibilización a padres y madres de familia, así como a docentes y profesionales que trabajan con niñas y niños.

- Integrar información y conocimientos básicos de alimentación, nutrición y adicciones, especialmente al tabaco, alcohol y otras drogas.

- Se requiere una revisión detallada de la prestación de servicios de salud dirigidos a las y los jóvenes, hay muchas experiencias y testimonios que mencionan malos tratos, sin información suficiente y clara, sin medicamentos, y muchas veces sin centros de salud y donde los hay, no son adecuados para zona rural y/o indígena

\section{Fuente: Elaboración propia.}

Mesa de trabajo 2: Violencias del sistema y feminicidios

El grupo de participantes de la segunda mesa de trabajo se centraron en el periodo del mes de enero a octubre del 2015, un año en el que se presentaron 7 casos de mujeres asesinadas (feminicidios), de los cuales la PGJEQ, solo reconoce como feminicidios dos (Gordillo, 2015). En esta mesa de trabajo, se presentaron tres testimonios más de jóvenes participantes en el Encuentro que no se han documentado. En consiguiente, las y los participantes de esta mesa de trabajo proponen lo presentado en la Tabla 2. 
Tabla 2. Recomendaciones en relación con la violencia del sistema y feminicidios

- Revisar el papel de las instituciones encargadas del Estado de prevenir y evitar la violencia y las desapariciones de mujeres y personas en general.

- Hacer investigaciones profundas y cuidadosas para indagar los motivos y las causas, respetando la dignidad y los derechos de la víctima, sus familiares y de los presuntos responsables.

- Impulsar y promover campañas amplias con acciones de convivencia.

- Fortalecer la sensibilización y capacitación de funcionarios para el respeto a los Derechos Humanos de todas las personas.

- Promover la participación en el entorno local y comunitario, proporcionando información sobre los Derechos Humanos y la importancia de su ejercicio, así como mantener la atención y la vigilancia para apoyar y documentar los casos de desapariciones de personas y violencias que se presenten.

- Promover muchas acciones educativas y campañas con "Educación crítica para desnaturalizar la violencia".

\section{Fuente: Elaboración propia.}

Mesa de Trabajo 3: Paternidad, familias y cuidado

El grupo de participantes de la tercera mesa de trabajo identificó varios problemas relacionados con las familias actuales. Estos incluyeron la sobrecarga del trabajo doméstico y de cuidados en las familias para las mujeres, lo cual les provoca estrés que en muchos casos se transforma en violencias hacia las niñas y los niños. Además, el grupo mencionó que el trabajo doméstico y de cuidados familiares no es valorado, ni se distribuyen sus responsabilidades entre todas las personas integrantes de la familia. Esta situación se refuerza con la marcada ausencia de la mayoría de los hombres, promovido por la exigencia social de alcanzar el rol de proveedor único. Por lo tanto, el grupo de esta mesa de trabajo propuso las siguientes recomendaciones expuestas en la Tabla 3.

Tabla 3. Recomendaciones en relación con la paternidad, familia y cuidados

- Sensibilizar y capacitar a hombres jóvenes para las tareas de cuidado y educación inicial de niñas y niños. Por ejemplo, hombres trabajando en guarderías y centros de desarrollo infantil.

- Motivar y promover la participación activa de todos los integrantes de una familia en el trabajo doméstico y los cuidados familiares.

- Cuestionar el uso excesivo de tecnología para ayudar a mejorar la comunicación en las familias.

- Reconocer la cotidiana transformación de las estructuras familiares, evitando la reproducción acrítica de la feminización de los cuidados.

- Documentar los beneficios psico-emocionales en niñas/os por la presencia respetuosa, solidaria, amorosa y acompañante de las y los adultos.

\section{Fuente: Elaboración propia.}

\section{Mesa de Trabajo 4: La diversidad sexual}

El grupo de participantes de la cuarta mesa de trabajo identificó que la discriminación por orientación sexual y/o identidad de género es el principal problema relacionado con la diversidad sexual. La discriminación es base para la exclusión, el estigma, la negación de Derechos y la violencia que se justifica en las familias, las instituciones y en la calle. Así, el grupo propuso las siguientes recomendaciones presentadas en la Tabla 4. 
Tabla 4. Recomendaciones en relación con la diversidad sexual

- Documentar los casos de discriminación y violencia a transexuales y transgénero y toda la comunidad LGBTTI.

- Ofrecer información suficiente y clara en torno a los Derechos Humanos para todas las personas, independientemente de su orientación sexo-genérica, edad, condición económica, origen étnico, alguna discapacidad, etc. para asumirlos, ejercerlos y defenderlos.

Fuente: Elaboración propia.

\section{Los materiales de la campaña}

Se concretaron tres carteles; uno sobre el rol de los hombres en el ámbito doméstico, el segundo nos habla de una forma sutil de violencia, como los piropos y el tercero muestra la riqueza de la diversidad, estos temas los entretejimos bajo el paradigma del cuidado (Gattino y Milesi, 2013), recuperando una expresión mexicana, contenida en la expresión "Fa xwi", que en ñhoñho significa "convidar", que refiere a una práctica de la tradición cultural mexicana de compartir, retomamos la expresión y construimos la frase "Compa, te convido a cuidarnos", como lema de esta campaña (véase Figura 1).

Fuente: Organización Salud y Género Querétaro A.C.
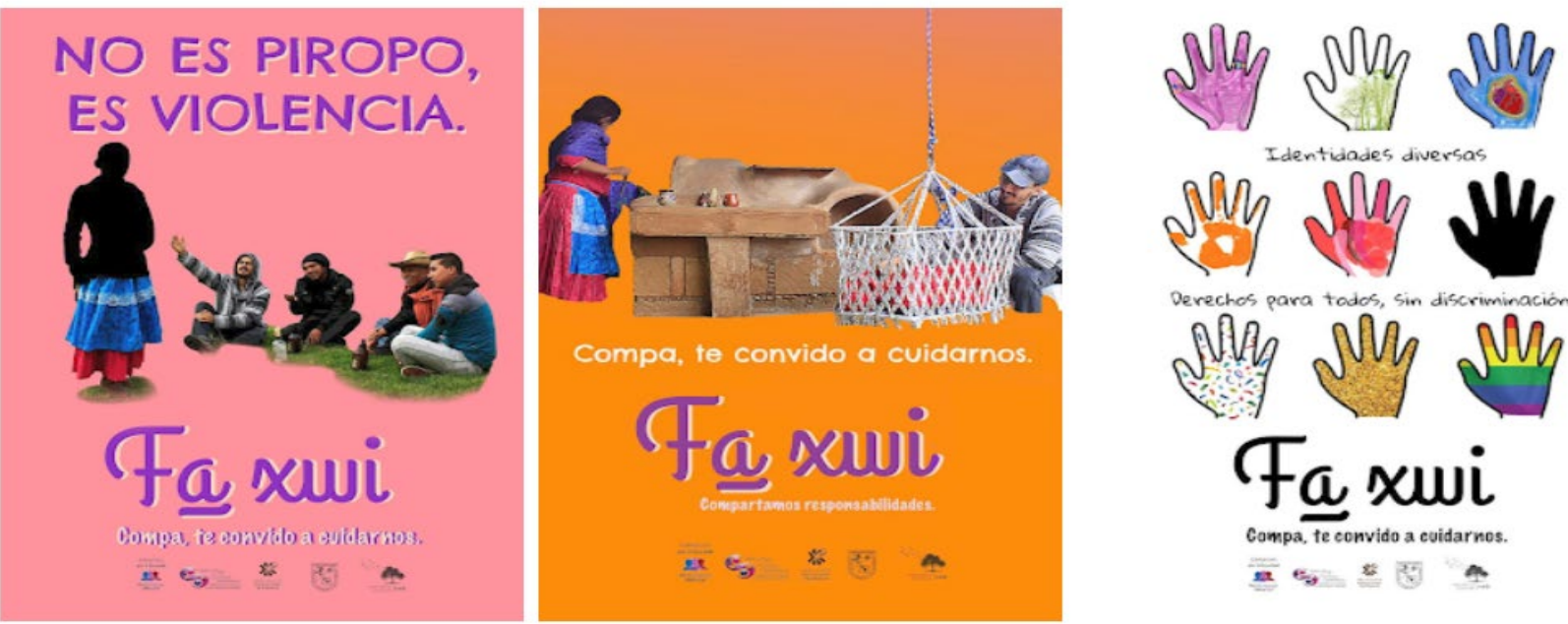

Figura 1. Ejemplares de carteles para la Campaña "Fa xwi: Te convido a cuidarnos".

\section{Los canales de difusión de la campaña}

Los spots de radio fueron producidos, grabados y difundidos en la radio universitaria, con la participación de cuatro jóvenes del Encuentro, entre ellas dos mujeres de origen Ñhoñho, quienes además hicieron la traducción a su idioma.

Por otra parte, la presentación pública se hizo aprovechando la invitación a las actividades del día internacional por la eliminación de la violencia contra las mujeres que se llevaría a cabo en la universidad, generando una actividad que denominaron
Conversatorio entre hombres ante la violencia hacia las mujeres.

Participaron cuatro hombres quienes a partir de su propia experiencia compartieron como es que fueron acercándose al feminismo, hasta convertirse en promotores de la igualdad sustantiva con las mujeres, además reflexionaron la importancia de ampliar y profundizar los estudios de género y las masculinidades, así como la responsabilidad de los hombres en la violencia machista y lo que se puede promover desde los propios hombres, presentando los carteles y los spots de radio. 


\section{Discusión}

a) Pertinencia de los resultados para dar respuesta a la problemática

La idea central que subyace en los hallazgos observados en los resolutivos de las mesas es el que tiene que ver con la urgencia de promover prácticas de autocuidado, tanto para las mujeres, como en los hombres, en virtud de que la socialización rígida de género sigue educando a las mujeres para atender las necesidades del "otro", mientras en los hombres, se les educa a esperar atenciones y cuidados de las mujeres. De tal manera que, ni mujeres, ni hombres desarrollan prácticas de autocuidado.

Esta ecuación es la base de una gran variedad de problemáticas que se van desarrollando a partir de esa manera de interactuar. Es decir, si modificamos la ecuación (promoción de prácticas de autocuidado), podemos presuponer que las interacciones entre mujeres y entre hombres, así como entre hombres y mujeres se den desde la autonomía y autodeterminación de cada persona, clave para la construcción de relaciones de mayor igualdad y menos violencias.

Es necesario el desarrollo de campañas de difusión y comunicación educativa en forma continua y permanente, al mismo tiempo que espacios de reflexión a través de talleres, con metodología participativa y vivencial, y adecuaciones culturales para favorecer el acceso a los servicios de salud para mujeres y hombres jóvenes.

El otro componente central que observamos es la promoción de la más amplia participación de las y los jóvenes en el diseño, el desarrollo y la evaluación de las campañas, así como de los talleres, pláticas, exposiciones, etc. En materia de Género, Derechos Humanos e interculturalidad, y no solo como depositarios y consumidores de las acciones educativas, es decir favorecer procesos de reconocimiento de las y los jóvenes como sujetos de su propio proceso de crecimiento y desarrollo. Esta información tiene el valor para contribuir en el futuro desarrollo de una agenda de juventudes y se presenta organizado de acuerdo con los temas de las mesas, identificando el/los principales problemas y sus correspondientes recomendaciones. b) Lecciones aprendidas sobre el desarrollo de la campaña

Como es el caso en cualquier acción comunitaria, la experiencia entorno al desarrollo de este proyecto ha generado varias lecciones de aprendizaje. Primero, la participación de hombres y mujeres jóvenes en el Encuentro Jóvenes, Derechos y Salud rebasó toda expectativa, misma que nos permite evidenciar la necesidad de trabajar en red desde lo local para apoyar la organización y participación comunitaria en la prevención y erradicación de la violencia machista y social (Haro y De Keijzer, 1998). Además, en la respuesta a la convocatoria de este evento observamos el gran interés que despierta el problema de la violencia ya sea por haberla experimentado de manera cercana y/o directa, por la difusión de los medios de comunicación masiva o por la emergencia de organizarse y accionar desde una educación crítica para desnaturalizar la violencia.

La pertinencia de motivar acciones de reflexión y diálogo con la participación de mujeres y hombres, que ayuden a desarrollar una perspectiva relacional, sin dejar de impulsar espacios específicos para mujeres y otros para hombres.

El éxito atribuido al proceso de desarrollo de la campaña de comunicación social se debe a los vínculos que se generaron entre las organizaciones con el apoyo de las redes sociales y las nuevas tecnologías. Así mismo, los autores creen que es de suma importancia que las y los jóvenes quienes participaron a lo largo del proyecto sigan generando vínculos con las instituciones educativas, así como con diversos organismos de la sociedad civil para incidir en las políticas públicas de igualdad de género que garanticen derechos sexuales y reproductivos de los y las jóvenes, así como la eliminación de las violencias.

La campaña: Fa xwi, Te convido a cuidarnos es un aporte de la comunidad Ñhoñho del Estado de Querétaro, que propone desnaturalizar la violencia hacia las mujeres desde un enfoque cultural y lenguaje relevante (Marín, 1993). Reflejado en los productos elaborados para la difusión amplia de la campaña, tales como los tres carteles y los spots de radio que muestran la riqueza y materialización de los diálogos y experiencias compartidas durante el 
diagnóstico participativo y las reuniones con la comunidad Ñhoñho posterior al Encuentro.

La difusión de una campaña de mercadeo social es de igual importancia que el desarrollo de sus materiales. En este caso particular, los cuatro spots de radio y una plataforma virtual construidos participativamente fueron el eje central para un ejercicio de comunicación educativa en dos contextos diferentes; jóvenes de la comunidad Ñhoñho y jóvenes de la comunidad universitaria, ambos del Estado de Querétaro.

\section{Referencias bibliográficas}

Arenas-Monreal, Luz; Jasso-Arenas, Jazmín y CamposNavarro, Roberto. (2011). Autocuidado: elementos para sus bases conceptuales. Global Health Promotion, 18(4), 42-48.

Barker, Gary y Verani, Fabio. (2008). La participación del hombre como padre en la región de Latinoamérica y el Caribe: una revisión de literatura crítica con consideraciones para políticas. Brasil: Instituto Promundo.

Boff, Leonardo. (2002). El Cuidado Esencial: Ética de lo humano, compasión por la tierra (Juan Valverde, Trad.). Madrid: Editorial Trotta, S.A. (Obra original publicada en 1999).

Bronfenbrenner, Urie. (1979). The ecology of human development: Experiments by nature and design. Cambridge: Harvard University Press.

Erikson, Erick. (1974). Identidad, juventud y crisis (Margarita Galeano, Trad.). Buenos Aires: Editorial Paidós. (Obra original publicada 1968).

Fransen-dos Santos, Raoul. (2009). Young people, sexual and reproductive health and HIV. Bulletin of the World Health Organization, 87(11), 877- 879. doi: 10.2471/BLT.08.059915.

Gattino, Silvia y Milesi, Andrea. (2013). Paradigma del cuidado: una nueva mirada para pensar las politicas de protección social y las estrategias de intervención. Villa María: Universidad Nacional de Villa María.

Gordillo, Mónica. (2015, 9 de octubre). Siete feminicidios consignados en el estado de Querétaro durante 2015. Revista Perfiles de Querétaro: Periodismo de Investigación. Recuperado de http://www.amqueretaro.com/queretaro/2015/10/09/7-feminicidios-consig- nados-en-el-estado-de-queretaro-durante-2015.

Haro, Jesús y De Keijzer, Benno. (Coords.). (1998). Participación comunitaria en salud: evaluación de experiencias y tareas para el futuro. Hermosillo, México: El Colegio de Sonora.

Instituto Nacional de Estadística y Geografía. (2013). Panorama de violencia contra las mujeres en México: ENDIREH 2011. México: INEGI.

Kiss, Adam; Ramon, S. y Sudres, Jean. (2004). L'investissement objectal et social de l'adolescent en rupture : étude clinique. Neuropsychiatrie de l'enfance et de l'adolescence, 52(1), 1-10.

López, María. (2013). Violencia contra las Mujeres en México: Tendencias Actuales. En ONU Mujeres, Seminario Internacional. Una Respuesta a la Violencia contra las Mujeres: La Convención de Belém de Pará. Recuperado de http://www. cndh.org.mx/sites/all/doc/programas/mujer/13 PromocionCapacitacion/13.3/G.pdf.

Marín, Gerardo. (1993). Defining culturally appropriate community interventions: Hispanics as a case study. Journal of Community Psychology, 21(2), 149-161.

Nastasi, Bonnie; Varjas, Kristen; Schensul, Stephen; Silva, Tudor; Schensul, Jean y Ratnayake, Priyani. (2000). The Participatory Intervention Model: A framework for conceptualizing and promoting intervention acceptability. School Psychology Quarterly, 15(2), 207-232.

ONU. (2015). Trends in Contraceptive Use Worldwide 2015. Nueva York: United Nations.

Promundo, Instituto PAPAI, Salud y Género y ECOS. (2013). Program HMD: A Toolkit for Action. Rio de Janeiro: Promundo.

Santos-Preciado, José; Villa-Barragán, Juan; GarcíaAvilés, Martha; León-Álvarez, Graciela; QuezadaBolaños, Sonia, y Tapia-Conyer, Roberto. (2003). La transición epidemiológica de las y los adolescentes en México. Salud Pública de México, 45(1), 140-152.

Servicios de Salud del Estado de Querétaro. (2010). Base de datos sobre Egresos Hospitalarios. Querétaro: SSEQ.

Sonfield, Adam. (2004). Meeting the Sexual and Reproductive Needs of Men Worldwide. The Guttmacher Report on Poblic Policy, 7(1), 9-12. Recuperado de https://www.guttmacher.org/sites/default/files/article files/gr070109.pdf. 